\title{
Prevención de la explotación sexual comercial infantil: experiencia a partir de la conformación de una comisión institucional de la UNED
}

\section{Susan Solís Rosales}

Magister Scientiae en Desarrollo Económico Local con énfasis en Turismo Comunitario de la Facultad Latinoamericana de Ciencias Sociales (FLACSO-CR) y el Instituto Tecnológico de Costa Rica (TEC). Actualmente directora de la Cátedra Turismo Sostenible de la Escuela de Ciencias Sociales y Humanidades de la UNED, Costa Rica.

Correo electrónico: ssolis@uned.ac.cr

\author{
Recibido: Enero 2014 • Aceptado: Agosto 2014
}

RESUMEN

El objetivo del presente trabajo es evidenciar las acciones y los esfuerzos que surgen a partir del compromiso y la convicción de ser, cada quien desde su ámbito de acción, parte de la lucha contra la explotación sexual comercial infantil, que violenta a nuestra niñez y adolescencia, así como dimensionar la función de las universidades ante dicha problemática. Se presenta el caso específico de la experiencia desarrollada por la Universidad Estatal a Distancia (UNED-CR) a partir de la conformación de una red organizativa denominada comisión institucional.

Palabras clave: explotación sexual, niñez, adolescencia, turismo, UNED.

\section{ABSTRACT}

The object of this work is to show the actions and efforts arising from the commitment and conviction of being, each one from its scope, part of the fight against these scourges, that violate our childhood and adolescence and dimensioning the role of universities to this problem. The specific case in the experience developed at University State to Distance (UNED-CR).

Key words: sexual explotation, childhood, adolescence, tourism, UNED.

Un agradecimiento a todas las personas que en la UNED han trabajado en este proyecto conjunto, desde la sede central hasta las diversas regiones del país. Un especial reconocimiento a la CONACOES; a la señora Elizabeth Ballestero, exsecretaria técnica, quien desde un inicio apoyó este proceso en la UNED, así como al Dr. Humberto Aguilar, director de la Escuela de Ciencias Sociales y Humanidades, al asignar recursos para los diversos proyectos y acciones que hasta la fecha se han desarrollado.

\section{Introducción}

En Costa Rica, la lucha contra la explotación sexual comercial infantil se inició en 1996, año en que se denunció y visibilizó el flagelo que atentaba contra nuestra niñez y adolescencia, el cual vino a dimensionarse con el desarrollo de la actividad turística: se promovían, de manera informal, redes inescrupulosas que promocionaban 
este como un país donde se podía realizar dichas prácticas sin ningún problema.

A partir de aquel momento, se creó la Comisión Nacional Contra la Explotación Sexual Comercial de Niños, Niñas y Adolescentes (CONACOES), adscrita al Patronato Nacional de la Infancia (PANI), órgano rector por parte del Estado en cuanto a la protección de las personas menores de edad (CONACOES, 2008). Su estructura organizativa se conformó con un plenario y tres subcomisiones (Prevención, Atención y Jurídica) y se desarrolló la parte técnica, la cual debía responder a los lineamientos que, en el plano político, dictara el Consejo Nacional de la Niñez y Adolescencia (CONNA).

En el 2007, se elaboró el Plan Nacional para la Erradicación de la Explotación Sexual Comercial de Niños, Niñas y Adolescentes 20082010. Su objetivo consiste en articular y definir acciones específicas según las competencias de las instancias que por su naturaleza deben ser partícipes activas en representación del Estado, así como aquellas invitadas al proceso; tal es el caso de universidades u organizaciones no gubernamentales (ONG).

En el 2011 por convenio internacional, se incorporaron los temas de la trata y el trabajo infantil como parte de la lucha para proteger a la niñez y a la adolescencia dentro del marco de la hoja de ruta diseñada para Costa Rica.

La Universidad Estatal a Distancia (UNED) de Costa Rica se incorporó a la Comisión Nacional en 2007; por su competencia se concentró en la Subcomisión de Prevención y fue representada por la Cátedra de Turismo Sostenible, al ser parte de la carrera de Gestión Turística Sostenible. El interés fue impulsar acciones y enfoques respetuosos de las poblaciones locales ante el desarrollo de las actividades turísticas, así como generar diversas actividades enfocadas hacia la capacitación para la prevención de esta problemática; ello de acuerdo con la misión de la UNED, como casa de educación superior, de atender a los problemas coyunturales de la sociedad según el contexto histórico y actual (UNED, 2005).
Por eso, resulta de interés evidenciar, en el plano institucional, los avances y los retos del tema de la niñez y la adolescencia en relación con la prevención de la explotación sexual comercial, la trata y el trabajo infantil en el contexto de una sociedad sumergida en una dinámica de consumo voraz, presa fácil para regirse por el mercado, elemento central del modelo de desarrollo neoliberal.

El objetivo del presente trabajo es evidenciar las acciones, los esfuerzos y los procesos básicos que surgen del compromiso y la convicción de ser, cada quien desde su ámbito de acción, parte de la lucha contra tales flagelos que violentan a nuestra niñez y adolescencia, así como dimensionar la función de las universidades ante dicha problemática.

Por consiguiente, el propósito es compartir la experiencia y lecciones aprendidas en un contexto de educación superior a distancia donde se evidencian los logros y desafíos por enfrentar en torno a la problemática.

\section{Antecedentes: ruta de organización interna de la UNED para el estudio y trabajo de la temática}

Con el fin de responder al reto de tratar el tema de la explotación sexual comercial infantil desde nuestra competencia como universidad, es decir, la inherencia en el ámbito educativo principalmente vía nuestro quehacer académico (docencia, extensión e investigación), se iniciaron las acciones y gestiones respectivas, como se detalla en el cuadro 1.

En el cuadro 1 se evidencia que, desde el proceso inicial, se gestionó la formalización de las acciones en aras de contar con los respaldos institucionales para la búsqueda de los recursos y el desarrollo de acciones específicas.

Por otra parte, cuando se hizo la convocatoria a las diversas instancias de la UNED para que formaran parte de la Comisión, hubo muy buena respuesta y hasta la fecha las integrantes activas de esta organización se mantienen, principalmente por su compromiso e identificación con la causa.

Revista ESPIGA Año XIV, N. ํ 29:71-80, enero-junio 2015 
CUADRO 1

Cronología de organización de la Comisión Institucional UNED

\begin{tabular}{lll}
\hline Año & \multicolumn{1}{c}{ Acción o gestión } & \multicolumn{1}{c}{ lnstancia que avala } \\
2007 & $\begin{array}{l}\text { Solicitud por parte de Ia ECSH de ingreso a la } \\
\text { CONACOES (participación sesión plenaria y } \\
\text { Subcomisión Prevención) }\end{array}$ & Consejo Nacional de la Niñez y Adolescencia. \\
2008 & $\begin{array}{l}\text { Aceptación formal de la UNED a la } \\
\text { CONACOES. }\end{array}$ & $\begin{array}{l}\text { Consejo Nacional de la Ninez y Adolescencia (se } \\
\text { emite acuerdo 03-04-08). }\end{array}$ \\
2009 & $\begin{array}{l}\text { Se oficializa, en el nivel institucional, la } \\
\text { representación en la CONACOES de la Escuela } \\
\text { de Ciencias Sociales y Humanidades a traves de } \\
\text { la Cátedra de Turismo Sostenible. También la } \\
\text { creación de la Comisión Institucional. }\end{array}$ & $\begin{array}{l}\text { Consejo Rectoría (CONRE Sesión 1602 2009, } \\
\text { art. 111, inciso 13), celebrada el 28 setiembre del }\end{array}$ \\
2010 & $\begin{array}{l}\text { Se conforma la Comisión Institucional para la } \\
\text { prevención de la explotación sexual comercial } \\
\text { infantil e inicia elaboración de plan de acción. }\end{array}$ & Adscrita a la Rectoría de la Universidad. \\
\hline 2010 & $\begin{array}{l}\text { Convocatoria y encuentro para la inserción de los } \\
\text { centros universitarios en las seis redes locales del } \\
\text { PANI. }\end{array}$ & $\begin{array}{l}\text { Comisión Institucional con la participación de la } \\
\text { CONACOES. }\end{array}$ \\
\hline $\begin{array}{l}\text { Finaliza Plan Estratégico de la Comisión } \\
\text { Institucional. Se elabora Plan de Acción } \\
\text { 2011-2014. }\end{array}$ & $\begin{array}{l}\text { Comisión Institucional, Centro de Planificación } \\
\text { Instional (CPPI). }\end{array}$ \\
\hline
\end{tabular}

Fuente: Susan Solís, a partir de informes y documento creación de la Comisión Institucional (2012).

La Comisión no cuenta con presupuesto asignado; por lo tanto, se promueve el aprovechamiento de algunos de los recursos con que cuentan las dependencias en ciertas partidas presupuestarias para integrar el eje de la temática.

Uno de los principales recursos de la Comisión han sido las personas que la integran, pues ellas movilizan los escasos recursos para la concreción del plan estratégico respectivo. En ese sentido, cumple un papel importante el capital social que conjuntan y generan al convocarse ante una problemática, una causa o un interés en común; según Long (2007), el capital social es la habilidad de una persona para movilizar los recursos que son de difícil acceso mediante el entramado de relaciones sociales, o bien, mediante la participación en redes o estructuras sociales particulares; dicho autor indica que los recursos no son solamente de tipo material, sino que pueden presentar características intangibles tales como "el conocimiento, habilidades, confianza, valores compartidos, principios organizacionales y representaciones" (2007: 253). Tales valores y habilidades han permitido generar en la Comisión un ambiente de confianza, vínculos recíprocos, lo mismo que compartir el conocimiento y la información entre las diversas dependencias representadas por personas muy comprometidas con la UNED y con la problemática.

Según el marco estratégico de la Comisión, su misión consiste en "planificar estrategias de prevención contra la explotación sexual comercial de trata y trabajo de niños, niñas y adolescentes, de acuerdo con las competencias institucionales y mediante la participación en redes interinstitucionales y la organización de espacios y actividades 
que permitan mitigar los factores de riesgo" (Centro de Planificación y Programación Institucional, CPPI, 2010: 1); asimismo, los valores que se definieron para desarrollar las acciones de la organización son ética, trabajo en equipo, innovación y diversidad en servicios.

Después de definir el marco estratégico de la Comisión, se establecieron las acciones que se desarrollarían y se plasmarían en un plan estratégico del 2011 al 2014, en el cual se contempla que cada año se realizaría un análisis y un informe de los avances, así como de las metas alcanzadas según lo propuesto en el plan.

La UNED cuenta con su representación en el nivel nacional al participar en la CONACOES; por su parte en el nivel institucional existen las dependencias ubicadas en la sede central (en Mercedes de Montes de Oca, San José); al mismo tiempo, participan aquellas regiones donde la Universidad tiene sus sedes y son consideradas prioritarias para la lucha contra la problemática, a saber, Los Chiles, Santa Cruz, Corredores,
Limón y Aguirre. Por ende, la UNED se encuentra representada en los cinco cantones, donde la administradora, o en su defecto algún funcionario asignado por parte del centro universitario, se integra a la red local de su zona para asistir a reuniones y definir acciones por desarrollar con sus comunidades desde la competencia como universidad.

\section{Aproximación conceptual}

De seguido, se presentan varios conceptos a propósito del tema que se estudia, en aras de puntualizar el estudio que se pretende desarrollar.

La CONACOES define explotación sexual comercial de niños y niñas adolescentes como un

"delito contra los derechos humanos de las personas menores de edad; consiste en la utilización de niños, niñas o adolescentes en actividades sexuales a cambio de una remuneración económica o cualquier otro tipo de retribución. Puede ser para tener relaciones

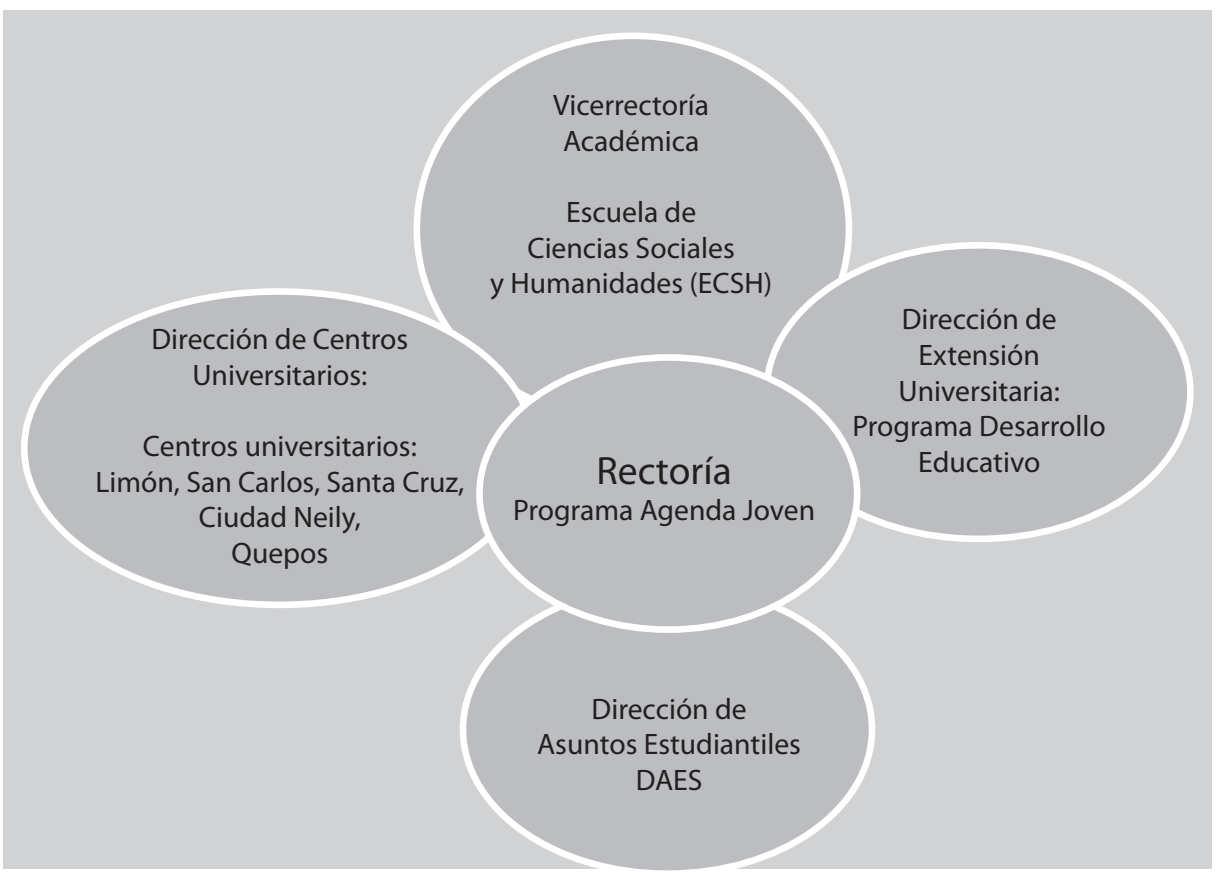

Figura 1. Conformación de la Comisión Institucional para la Prevención de la Explotación Sexual Comercial de Niños, Niñas y Adolescentes. Fuente: Susan Solís, a partir del documento creación Comisión Institucional (2012). 
CUADRO 2

Porcentaje de personas que no harían nada ante una situación de explotación sexual comercial, por país. Datos comparativos años 2005 y 2008.

\begin{tabular}{cccccccc} 
País / Año & Guatemala & El Salvador & Honduras & Nicaragua & Costa Rica & Panamá & Rep. Dominicana \\
2005 & 52,1 & 39,3 & 39,1 & 36,7 & 50,1 & 17 & 43,1 \\
2008 & 51,9 & 77,4 & 49,9 & 40,6 & 40,8 & 25,9 & 47,7 \\
\hline
\end{tabular}

Fuente: Susan Solís, reconfiguración a partir de la tabla 3. Porcentaje de personas que no harían nada ante una situación de explotación sexual comercial, por país y sexo. Datos comparativos años 2005 y 2008. OIT-IPEC (2009:35).

sexuales, para tomar fotos de contenido sexual o erótico, hacerles tomas para películas pornográficas o en su utilización en espectáculos sexuales, exponerles actividades sexuales entre adultos" (2010: 1).

El concepto anterior funciona como un referente en el ámbito nacional, pues es acuñado por el ente rector en materia de niñez y adolescencia costarricense, el Patronato Nacional de la Infancia (PANI).

Azaola y Estes (2003: 31) establecen una definición sobre explotación sexual comercial de niños que recoge la esencia de la Convención de los Derechos del Niño y amplía en otros aspectos:

Abuso sexual de niños que involucra ventajas financieras para una o varias de las partes que intervienen en la actividad sexual y que puede ser de dos tipos: 1) transferencia de dinero de un adulto a un niño en intercambio por sexo y 2) provisión en especies o servicios que un adulto intercambia por sexo con un niño (i.e. casa, comida, protección, etc).

Una característica de tal problemática radica en su origen multicausal, es decir, hay una serie de factores que le dan complejidad. Usualmente se determina en el entorno más próximo de los niños y adolescentes, con la participación de su propia familia o amigos muy cercanos a este círculo (Claramunt, 2002; Azaola y Estes, 2003; Sanabria, 2004). Aunado a ello está la falta de oportunidades, de educación, así como la pobreza y una cultura de consumo que presiona a la persona a obtener las cosas sin importar el costo con tal de ser integrada a su grupo. Estos, entre otros aspectos, crean ambientes propicios para la violación de los derechos de las personas menores de edad.

La situación anterior demuestra la complejidad de esta problemática social. Por ende, requiere una fuerte articulación del tejido institucional existente en un país (Claramunt, 2002), pues el Estado es el principal actor en la generación de esta dinámica (Kliksberg, 1998) para proteger los derechos humanos de los habitantes; pero todos $\mathrm{y}$ todas sin distinción somos parte de esta lucha.

En ese sentido, es preocupante la posible actitud de nuestra sociedad ante la problemática, según un estudio realizado por la OIT (2009b).

Según dicho estudio, un $97,2 \%$ de los costarricenses identifica que la explotación sexual es un delito. Sin embargo, el cuadro 2 muestra que el $40,7 \%$ indica que no denunciaría en caso de enterarse de alguna situación de explotación; tal población externa que "no le importa o no le interesa"; además, se demuestra una gran desconfianza hacia las instituciones encargadas de castigar a los explotadores o personas involucradas en este tipo de delitos.

Se trata de datos reveladores, que constituyen un llamado de atención para ampliar los niveles de sensibilización de la población, a fin de que el tema no sea percibido con tal indiferencia o de que estas prácticas no se sigan institucionalizando en la cultura, pues perjudican el sano desarrollo de una sociedad.

\section{Principales logros}

El trabajo en equipo y el compromiso de las dependencias cuyas integrantes conforman la Comisión Institucional, han hecho posible 
alcanzar diversos logros, los cuales pueden ser identificados de forma directa o indirecta mediante listas de asistencia, minutas de reuniones y retroalimentación por parte de las integrantes. Los logros se han gestionado en el marco de la Comisión, o bien, como resultado de la motivación de cada persona desde su ámbito de acción para ser parte del proceso de la lucha contra la explotación sexual comercial infantil.

Entre los principales avances sobre el tema en el ámbito institucional, se pueden mencionar los siguientes:

- La conformación y formalización de la Comisión Institucional, que facilitó la creación de un espacio de búsqueda de recursos y desarrollo de acciones concretas, sobre la base de vinculaciones entre las diversas instancias universitarias $u$ otros organismos gubernamentales y no gubernamentales.

- Representación en la CONACOES, espacio en el ámbito nacional que permite formalizar los contactos con las instituciones del Estado, así como en diversas ONG que están trabajando el tema; de igual forma, se desarrollan acciones y retroalimentación del quehacer de cada entidad. En este marco, se participó en la elaboración de los siguientes documentos: Plan Nacional contra la Explotación Sexual Comercial de Niñas, Niños y Adolescentes en Costa Rica 2008-2010 y Compendio de Normas: Normativa sobre Explotación Sexual Comercial de Niños, Niñas y Adolescentes, las cuales orientan a la sociedad civil sobre las instancias estatales a que puede acudir si detecta casos de explotación sexual.

- Articulación de acciones y proyectos con las diversas instancias universitarias, mediante las reuniones mensuales que se calendarizan a lo largo de todo el año con el objetivo de asegurar la asistencia de las partes integrantes de la Comisión.

- Representación en las redes locales de prevención de la explotación sexual comercial en los cantones que cuenten con sedes de la UNED y que de igual forma son miembros activos de la Comisión Institucional. Los centros universitarios de Limón (representado por su administradora, Lidia Urbina, y la asistente Marilyn Sánchez), Santa Cruz (cuya administradora es Lúcida Guevara) y San Carlos (con la participación de la encargada de Extensión Universitaria Lucrecia Córdoba) reportaron una inserción en la red de forma activa y continua mediante las actividades de capacitación y vinculación con las instituciones representadas en sus respectivas comunidades, actividades reportadas y evidenciadas en sus planes de acción local.

- Espacio de reflexión a propósito de las lecciones aprendidas y los retos en cuanto el proceso de ejecución y las acciones de las redes locales mediante la organización de videoconferencias por parte de los centros universitarios de Limón, Santa Cruz y San Carlos (Los Chiles).

- Capacitaciones referidas a la materia y los campos de acción de las partes integrantes de la Comisión, a partir de talleres previos que determinaron los temas de interés (se contactó a especialistas para abordar dichos contenidos) ${ }^{1}$.

- Inclusión de la UNED como parte del informe que, en el ámbito costarricense, debe presentarse en relación con el avance de la lucha contra la explotación sexual comercial (Ballestero, 2011).

- Divulgación, por los diversos medios de comunicación (internos y externos a la UNED), de las acciones ejecutadas en cuanto a la temática por parte de la Universidad, al igual que espacios formalizados de toma de decisiones y acuerdos, como el caso de consejos de Escuela y la Dirección de Centros Universitarios.

- Otra de las acciones lideradas por la Escuela de Ciencias Sociales y Humanidades (ECSH), promovida inicialmente por la

1. Para más detalles sobre las actividades desarrolladas por parte de las integrantes de la Comisión Institucional, se sugiere ingresar al blog <esasuntodetodos.com>. 
Cátedra de Psicología Educativa, consiste en un proyecto denominado Intercátedras. $\mathrm{Su}$ objetivo es realizar una vinculación interdisciplinaria y el quehacer académico (docencia, extensión e investigación) para abordar la temática de la explotación sexual comercial infantil de manera integral. Ana Lorena Vargas, actual coordinadora del Proyecto, indicó lo siguiente:

desde la ECSH se ha tratado de abordar el tema, con el fin de colaborar con las políticas públicas del país y así promover la concienciación y sensibilización, no solo de los adultos, sino también de los niños y jóvenes... (Acontecer, 2012, 11 octubre).

- El procedimiento utilizado es que cada encargado de cátedra valora o selecciona el cuatrimestre en que participará, así como el curso o los cursos y las actividades didácticas y pedagógicas que implementará para desarrollar la problemática con los estudiantes matriculados. En reuniones de cátedra, se discute conjuntamente con las tutoras y los tutores los elementos fundamentales de las actividades y se eligen al final los trabajos estudiantiles que demostraron cumplir mayormente con los criterios que la cátedra estableció para el desarrollo de las actividades evaluativas o de formación. Tales proyectos o investigaciones se presentan en la actividad que se celebra cada setiembre en el Día para la Prevención y Erradicación de la Explotación Sexual Comercial Infantil. Cada año el grupo que conforma el Proyecto Intercátedras se plantea las nuevas temáticas por trabajar y que afecten a la niñez y adolescencia, siempre enfocadas en el marco de los derechos humanos.

La ECSH cuenta con 28 cátedras, de las cuales 18 integran dicho Proyecto, es decir, el 64\% de estas y además una encargada de carrera, quien tiene como recargo los cursos de la carrera. De igual forma, se unen al proyecto el Programa de Extensión de la Escuela de Ciencias Sociales y la plataforma radiofónica Onda UNED. ${ }^{2}$
No obstante, en los procesos de capacitación así como otros espacios de reflexión, se ha comentado que esta problemática social "es asuntos de todos" y que en cualquier momento todos aquellos recursos humanos y tecnológicos que se quieran sumar a la lucha contra la explotación sexual comercial infantil lo pueden hacer.

Tal es el caso de las herramientas tecnológicas que se utilizan para canalizar el mensaje preventivo de dicho flagelo. En este sentido, se diseñó un blog denominado "Es asuntos de todos"; cada participante del proyecto aportó material o puntos de vista desde su disciplina; el blog recibe una muy buena visitación. Se dispuso también de la plataforma de radio Onda UNED para presentar los contenidos o los temas de estudio en una forma más dinámica y didáctica. Ambos recursos han permitido el acceso a un universo importante de personas, ya sean estudiantes de la Universidad o externas a la institución.

En el cuadro 3, se muestra la incidencia de la iniciativa en la sensibilización hacia dicha problemática en aras de cambiar o revalorar la actitud de tolerancia que al inicio del artículo se comentaba. El solo hecho de entrar en un proceso de reflexión y provocar un diálogo entre nuestros estudiantes, que son o serán en un futuro profesionales, abona a su formación integral como seres humanos.

Entonces, no se enfoca el proceso en formar o capacitar a profesionales para que se inserten en los procesos productivos del país sin contemplar otros valores que de igual forma son fundamentales, pero que en nuestra sociedad no se fomentan, posiblemente a causa de un modelo de desarrollo neoliberal que incentiva la generación de riqueza material, es decir, el tener más que el ser. Así se resta valor a iniciativas y proyectos

2. Onda UNED es una plataforma radiofónica mediante la cual se abordan diversas temáticas de interés para la comunidad universitaria. Se puede consultar las producciones realizadas sobre el tema ingresando a www.ondauned.com, en la sección Series Especiales, en Proyecto Intercátedras: Explotación Sexual. 
CUADRO 3

Participación en el Proyecto Intercátedras según cátedras y número de estudiantes

\begin{tabular}{|c|c|c|c|c|c|}
\hline \multirow[t]{2}{*}{ Cátedra- Programa } & \multicolumn{4}{|c|}{ Año de participación } & \multirow[t]{2}{*}{ Estudiantes matriculados } \\
\hline & 2010 & 2011 & 2012 & 2013 & \\
\hline Artes & & & $\mathrm{x}$ & $\mathrm{x}$ & $\begin{array}{l}\text { diseño y seguimiento de blog } \\
\text { con una visitación de } 3000 \text { personas }\end{array}$ \\
\hline Ciencias Penales & & $\mathrm{x}$ & $\mathrm{x}$ & $\mathrm{x}$ & 60 \\
\hline Criminología & $\mathrm{x}$ & & $\mathrm{x}$ & $\mathrm{x}$ & 70 \\
\hline Derecho & & & & $\mathrm{x}$ & 100 \\
\hline Enseñanza del Inglés & & & & $\mathrm{x}$ & $40-50$ \\
\hline Extensionista de ECSH & $\mathrm{x}$ & $\mathrm{x}$ & $\mathrm{x}$ & $\mathrm{x}$ & logística y divulgación \\
\hline Formación Cívica y Geográfica & $\mathrm{x}$ & $\mathrm{x}$ & $\mathrm{x}$ & $\mathrm{x}$ & $50-80$ \\
\hline Gramática y Literatura & & & $\mathrm{x}$ & $\mathrm{x}$ & $150-450$ \\
\hline Historia & $\mathrm{x}$ & $\mathrm{x}$ & $\mathrm{x}$ & $\mathrm{x}$ & $800-2000$ \\
\hline Métodos y Técnicas & & & $\mathrm{x}$ & & 2550 \\
\hline $\begin{array}{l}\text { Programa de Comunicación (Radio) } \\
\text { Onda UNED }\end{array}$ & $\mathrm{x}$ & $\mathrm{x}$ & $\mathrm{x}$ & $\mathrm{X}$ & $\begin{array}{l}23 \text { producciones con una } \\
\text { visitación de } 10006 \text { personas }\end{array}$ \\
\hline Programa Gestión Secretarial de Oficinas & & & $\mathrm{x}$ & $\mathrm{x}$ & $100-120$ \\
\hline Psicología & & & $\mathrm{x}$ & & 50 \\
\hline Psicología Educativa & $\mathrm{x}$ & $\mathrm{x}$ & $\mathrm{x}$ & $\mathrm{x}$ & 350 \\
\hline Psicopedagogía & & & $\mathrm{x}$ & $\mathrm{x}$ & 90 \\
\hline Teología & & $\mathrm{x}$ & $\mathrm{x}$ & $\mathrm{x}$ & 20 \\
\hline Trabajo Social & $\mathrm{x}$ & $\mathrm{x}$ & $\mathrm{x}$ & $\mathrm{x}$ & $50-200$ \\
\hline Turismo Sostenible & $\mathrm{x}$ & $\mathrm{x}$ & & & $80-120$ \\
\hline
\end{tabular}

Fuente: Susan Solís, a partir de las entrevistas a informantes clave que participaron del Proyecto Intercátedras entre los períodos del 2010-2013 (2013).

como este, pues el modelo neoliberal apuesta todos sus recursos a la generación de más riqueza.

Resulta significativo el número de estudiantes (determinado según matrícula) que están abordando el tema; a ello se añade el hecho de que algunas de esas asignaturas se ofertan en varios cuatrimestres al año. Se multiplican, entonces, los estudiantes que tendrán acceso al tema y de una forma muy mediada con las propuestas de las diversas cátedras.

Según las personas encargadas de cátedra y la encargada de programa, la experiencia del proyecto ha sido muy gratificante para sus tutores y estudiantes, ya que el plantear el tema en los diversos cursos les ha permitido conocer más la problemática y confrontar mitos que la sociedad ha creado respecto de esta; asimismo, logran una mayor sensibilización, a la vez que se les despierta un interés por investigar más el tema y confrontar el miedo a denunciar casos. Se determinaron también hechos relevantes como, por ejemplo, que en algunos centros educativos no estaba clara la problemática ni cómo se activan los protocolos de atención.

Por otra parte, se identificó que este tipo de explotación presenta una connotación muy diferente según la región del país, al existir diversos elementos culturales; pero, finalmente, siempre es una violación de los derechos de niños, niñas y adolescentes. Por eso, resulta fundamental conocer bien el concepto para no aceptar prácticas que culturalmente se han presentado como tolerables. 


\section{Retos institucionales para el desarrollo de la problemática}

Los desafíos que enfrenta la UNED para continuar atendiendo esta problemática se pueden enfocar en tres dimensiones, según el criterio de las personas miembros de esta red organizativa denominada Comisión:

- Establecimiento de una política institucional referida a la niñez y la adolescencia, que permita robustecer los proyectos y las acciones que en el presente artículo se han expuesto y que se convierten en importantes plataformas para la generación de lecciones. Se sabe que estos grupos sociales constantemente sufren la violación de sus derechos, no solamente ante la problemática de la explotación sexual comercial, sino también mediante otras formas que las redes criminales o los procesos culturales locales institucionalizan, como la trata de personas y el trabajo infantil.

- Diálogo a lo interno de la Universidad para reflexionar sobre el posicionamiento epistemológico, al igual que los supuestos teóricos por los que la UNED asumiría estas problemáticas que afectan a nuestra niñez y adolescencia. Es fundamental incentivar la investigación académica sobre el tema y de ese modo generar una fuente preponderante para dicha discusión; en esa línea, es posible la definición del rumbo por seguir en el nivel institucional según el modelo pedagógico y la consecución de recursos.

- Mantener la apertura de los canales de comunicación y la continua búsqueda de prácticas y mecanismos que promuevan la articulación entre las diversas dependencias de la UNED, tanto las ubicadas en la sede central como en las regiones. Es de gran relevancia este vínculo, ya que permite la realimentación desde la realidad de estos contextos, y facilita la inserción al territorio con proyectos o propuestas orientadas según requerimientos más puntuales y reales. Y, por supuesto, se promueve la riqueza de conocimiento y aprendizaje que se ha generado al conformarse verdaderos equipos de trabajo en pro de la niñez y adolescencia, sin importar las distancias o contextos culturales.

\section{Reflexión final}

El proceso realizado por la UNED puede considerarse como una buena práctica en el nivel institucional. Además se evidenció su trascendencia a espacios informales; tal es el caso de la representación de la UNED en las regiones y su inserción en las dinámicas locales, así como el porcentaje de participación constante en el Proyecto Intercátedras por parte de la cátedras y programas, iniciativa donde se exalta la vinculación interdisciplinaria y la participación del estudiantado para promover la prevención de dicha problemática.

$\mathrm{Al}$ respecto se puede indicar, primero, que el dialogo participativo de los diversos actores de las regiones y la sede central permitió concretar acciones que influyeron en las localidades con presencia física de la UNED y en nuestras poblaciones estudiantiles ubicadas a lo largo y ancho del país. Los diversos actores sociales se constituyen en agentes multiplicadores de los conocimientos o reflexiones acerca de los contenidos temáticos. Y, segundo, en el ámbito académico, resulta importante aprovechar la experiencia generada por el Proyecto Intercátedras para analizar la sostenibilidad del estudio de la temática de niñez y adolescencia, así como otras temáticas que a futuro surjan según las dinámicas sociales, en aras de incursionar de forma permanente en los diseños curriculares de diversos planes de estudios; además, para efectos de futuras líneas de investigación, se podría retomar el análisis a profundidad del tratamiento que cada disciplina o asignatura ha realizado en relación con la temática de los derechos de la niñez y la adolescencia para ampliar el ámbito de conocimiento al respecto.

También es fundamental exaltar las capacidades de movilización de recursos (locales y regionales) que caracterizan a las personas miembros de la red organizativa, estableciendo una 
importante cohesión de grupo para alcanzar los objetivos y metas propuestas.

Se observa la influencia que la educación -y en este caso particular, las universidades- tiene sobre la sociedad, al menos desde el ámbito de la prevención, para erradicar estas problemáticas que violentan los derechos humanos.

En la sociedad actual, prima el desarrollo tecnológico: el ser humano en muchas ocasiones se determina como una pieza más del proceso. No obstante, el personal de la UNED ha querido demostrar que existen otras formas de alcanzar un desarrollo con rostro humano.

La tarea es ardua, pues aún en nuestra institución la lucha por los recursos se mantiene. Por eso, es primordial promover la incidencia en el nivel político institucional, ya que si bien es cierto se demuestra la apertura y el compromiso de las diversas instancias, aún se sigue determinando resistencia o, en su defecto, promesas que quedan en los discursos pero a la hora de ejecutar acciones se disipa el contenido presupuestario se aduce que se está en época de crisis.

Por otra parte, los diversos espacios de reflexión y discusión determinan que debe evolucionar la estructura que inicialmente se conformó como una comisión institucional para la prevención de la explotación sexual, la trata y el trabajo infantil. Es decir, la UNED en el nivel políticoinstitucional debe adoptar una postura más enfática acerca de cómo abordará las problemáticas que afectan a nuestra niñez y adolescencia, tomando en cuenta el contexto actual, el sistema de educación a distancia de la Universidad y, por supuesto, visualizando el futuro.

Acerca de esta experiencia que se ha compartido es posible puntos de mejora, al igual que se rescata el intercambio con otras instancias $u$ organizaciones que deseen transitar por la ruta de la prevención y formación sobre esta temática.

\section{Referencias}

Acontecer (2012, 11 octubre). Proyecto Intercátedras trabaja para mitigar explotación sexual comercial. Recuperado de http://www.uned.ac.cr/acontecer/index. php?option $=$ com_content $\&$ view $=$ article $\&$ id $=156$ 9:proyecto-intercatedras-trabajaparamitigarexplotaci onesxualcomercial $\&$ catid $=48$ : ociedad $\&$ Itemid $=80$

Azaola, Elena y Estes, Richard J. (2003). La infancia como mercancía sexual. México, Cánada, Estados Unidos. Coyoacán: Siglo XXI Editores.

BAllestero, Elizabeth (2011). Prácticas integrales innovadoras para la garantía y protección integral de los derechos adolescentes en situaciones explotación sexual comercial. Recuperado de https//www.google.co.cr/ \#q=elizab eth+ballestero+buenas + pr $\% \mathrm{C} 3 \% \mathrm{~A} 1$ cticas + explotaci $\%$ $\mathrm{C} 3 \% \mathrm{~B} 3 \mathrm{n}+$ sexual + comercial+pani + costa + rica

Centro de Planificación y Programación Institucional, CPPI (2011). Propuesta para la creación de la Comisión Institucional para la Prevención de la Explotación Sexual Comercial en Niños, Niñas y Adolescentes. San José: UNED.

Claramunt, María Cecilia (2002). Costa Rica, explotación sexual comercial de personas menores de edad: una evaluación rápida. Ginebra: OIT-IPEC.

Comisión Institucional para la Prevención de la Explotación Sexual Comercial (2012). Informe Ejecutivo de las acciones 2011-2012. San José: UNED.

CONACOES (2008). Plan Nacional contra la Explotación Sexual Comercial de Niñas, Niños y Adolescentes en Costa Rica 2008-2010. San José: IPEC-OIT.

CONACOES (2010). Material de divulgación e información acerca de la prevención de la explotación sexual comercial infantil. S. e.

KLIKSBERG, Bernardo (1998). Repensando el Estado para el desarrollo social: más allá de dogmas y convencionalismos. San José: UCR.

Long, Norman (2007). Sociología del desarrollo: una perspectiva centrada en el actor. México: El Colegio de San Luis.

OIT (2009a). Contribución a la prevención y eliminación de la explotación sexual comercial en América Central y República Dominicana. ; Ya es hora! (8). San José: OIT.

OIT (2009b). El comercio sexual con personas menores de edad en Centroamérica, Panamá y República Dominicana. Segundo Estudio de Tolerancia social. Análisis de Resultados, Desafios y Recomendaciones. San José: OIT.

SAnABria, Jorge R. (2004). Autonomía y prospección en adolescencia víctimas de explotación sexual. Cuaderno de Ciencias Sociales. San José: Facultad de Ciencias Sociales (FLACSO).

Universidad Estatal a Distancia (2005). Creación y organización de la UNED. San José: EUNED. 